\title{
A FAMILY OF BELL TRANSFORMATIONS
}

\author{
DANIEL BIRMAJER, JUAN B. GIL, AND MICHAEL D. WEINER
}

\begin{abstract}
We introduce a family of sequence transformations, defined via partial Bell polynomials, that may be used for a systematic study of a wide variety of problems in enumerative combinatorics. This family includes some of the transformations listed in the paper by Bernstein \& Sloane, now seen as transformations under the umbrella of partial Bell polynomials. Our goal is to describe these transformations from the algebraic and combinatorial points of view. We provide functional equations satisfied by the generating functions, derive inverse relations, and give a convolution formula. While the full range of applications remains unexplored, in this paper we show a glimpse of the versatility of Bell transformations by discussing the enumeration of several combinatorial configurations, including rational Dyck paths, rooted planar maps, and certain classes of permutations.
\end{abstract}

\section{INTRODUCTION}

Aiming at developing a unifying approach for a variety of enumeration problems, and in the spirit of the work by E. T. Bell on partition polynomials, in this paper we introduce a family of sequence transformations defined via partial Bell polynomials.

Let $a, b, c, d$ be fixed numbers. Given a sequence $x=\left(x_{n}\right)_{n \in \mathbb{N}}$, we let $y=\mathscr{Y}_{a, b, c, d}(x)$ be the sequence defined by

$$
y_{n}=\sum_{k=1}^{n} \frac{1}{n !}\left[\prod_{j=1}^{k-1}(a n+b k+c j+d)\right] B_{n, k}\left(1 ! x_{1}, 2 ! x_{2}, \ldots\right) \text { for } n \geq 1,
$$

where $B_{n, k}$ denotes the $(n, k)$-th (exponential) partial Bell polynomial. We call $\mathscr{Y}_{a, b, c, d}(x)$ the BELL transform of $x$ with parameters $(a, b, c, d)$.

For $k=0,1,2, \ldots$, the polynomials $B_{n, k}\left(z_{1}, \ldots, z_{n-k+1}\right)$ may be defined through the series expansion

$$
\frac{1}{k !}\left(\sum_{j=1}^{\infty} z_{j} \frac{t^{j}}{j !}\right)^{k}=\sum_{n=k}^{\infty} B_{n, k}\left(z_{1}, z_{2}, \ldots\right) \frac{t^{n}}{n !} .
$$

These polynomials are homogeneous of degree $k$, of weight $n$, and they can be written as

$$
B_{n, k}\left(z_{1}, \ldots, z_{n-k+1}\right)=\sum_{\alpha \in \pi(n, k)} \frac{n !}{\alpha_{1} ! \alpha_{2} ! \cdots}\left(\frac{z_{1}}{1 !}\right)^{\alpha_{1}}\left(\frac{z_{2}}{2 !}\right)^{\alpha_{2}} \cdots,
$$

where $\pi(n, k)$ denotes the set of multi-indices $\alpha \in \mathbb{N}_{0}^{n-k+1}$ such that

$$
\alpha_{1}+\alpha_{2}+\cdots=k \text { and } \alpha_{1}+2 \alpha_{2}+3 \alpha_{3}+\cdots=n .
$$

Note that $B_{n, k}$ contains as many monomials as the number of partitions of $[n]=\{1, \ldots, n\}$ into $k$ parts. Thus, if $x$ enumerates some class of building blocks (with $x_{j}$ distinct blocks of type $j$ ), then the sequence $\mathscr{Y}_{a, b, c, d}(x)$ counts the number of objects that can be made 
from these building blocks by placing them (according to their type) on a set of partitions induced by the parameters $(a, b, c, d)$. Moreover, the term

$$
\frac{1}{n !}\left[\prod_{j=1}^{k-1}(a n+b k+c j+d)\right] B_{n, k}\left(1 ! x_{1}, 2 ! x_{2}, \ldots\right)
$$

gives the number of such objects made with exactly $k$ blocks. For example, if the induced partitions consist of interval blocks, then the set of resulting objects of length $n$ made with $k$ such blocks is given by

$$
\frac{k !}{n !} B_{n, k}\left(1 ! x_{1}, 2 ! x_{2}, \ldots\right)
$$

This corresponds to $(a, b, c, d)=(0,1,-1,1)$. Moreover, the sum over $k$ from 1 to $n$ gives the known INVERT transform of $x$, see e.g. [2, 4, 14, and (2) may be interpreted as the number of colored compositions of $n$ with $k$ parts, where part $j$ comes in $x_{j}$ different colors.

Another special case of broad interest is the NONCROSSING PARTITION transform, introduced by Beissinger in [2] and systematically studied by Callan in [13. It corresponds to the parameters $(a, b, c, d)=(1,0,-1,1)$, giving $\prod_{j=1}^{k-1}(a n+b k+c j+d)=\frac{n !}{(n-k+1) !}$. In this case, (11) becomes

$$
y_{n}=\sum_{k=1}^{n} \frac{1}{(n-k+1) !} B_{n, k}\left(1 ! x_{1}, 2 ! x_{2}, \ldots\right),
$$

which counts the configurations obtained by placing the building blocks enumerated by $x$ on top of the noncrossing partitions of $[n]$. In particular, if $x$ is the sequence of ones, denoted by $\mathbb{1}=(1,1, \ldots)$, then

$$
\begin{aligned}
y_{n} & =\sum_{k=1}^{n} \frac{1}{(n-k+1) !} B_{n, k}(1 !, 2 !, \ldots) \\
& =\sum_{k=1}^{n} \frac{1}{(n-k+1) !} \frac{n !}{k !}\left(\begin{array}{c}
n-1 \\
k-1
\end{array}\right) \\
& =\sum_{k=1}^{n} \frac{1}{n}\left(\begin{array}{c}
n \\
n-k
\end{array}\right)\left(\begin{array}{c}
n \\
k-1
\end{array}\right)=\frac{1}{n}\left(\begin{array}{c}
2 n \\
n-1
\end{array}\right)=\frac{1}{n+1}\left(\begin{array}{c}
2 n \\
n
\end{array}\right) .
\end{aligned}
$$

Thus $\mathscr{Y}_{1,0,-1,1}(\mathbb{1})$ is the sequence of Catalan numbers that enumerates noncrossing partitions, Dyck paths, rooted trees, and many others combinatorial objects (see e.g. [36]).

The family $\mathscr{Y}_{a, b, c, d}$ also includes several of the known transformations studied by Bernstein and Sloane [4]. For example, EXP, REVERT, and CONV can be realized as instances of BELL.

Our goal is to study $\mathscr{Y}_{a, b, c, d}$ from the algebraic and combinatorial points of view. In Section 2 we give explicit formulas for the inverse $\mathscr{Y}_{a, b, c, d}^{-1}$. In Section 3 , we provide equivalent forms of (10) in terms of generating functions. The results in these two sections are obtained using Lagrange inversion together with certain interpolating properties of the partial Bell polynomials proved in [6].

In Section 4 we prove a convolution formula for BeLL transforms of the form $\mathscr{Y}_{a, b, c, 1}$, and we give a recurrence relation for $\mathscr{Y}_{a, b,-1,1}$ assuming that $a$ and $b$ are not both equal to 0 . Section 5 is a compilation of basic examples that showcase special instances of $\mathscr{Y}_{a, b, c, d}$. 
In Section 6 we discuss combinatorial applications and give some examples that illustrate how $\mathscr{Y}_{a, b, c, d}$ may be used to link the enumeration of certain classes of combinatorial structures with the enumeration of building blocks that serve as "primitive elements" within each class. In that section, we will focus on rational Dyck paths, rooted planar maps, and certain classes of permutations. For a general approach to the enumeration of irreducible combinatorial objects, we refer to the work by Beissinger [2].

It is not surprising that the results of this paper heavily rely on properties of the partial Bell polynomials. For a list of basic properties and combinatorial identities, we refer the reader to $[3,15,18,19,22,29,30,31,38$, and the references therein. In an attempt to make the paper more readable and as self-contained as possible, we have included an appendix with the main technical results on partial Bell polynomials used for our arguments.

\section{INVERSE RELATIONS}

We start by proving an interpolating relation for $y=\mathscr{Y}_{a, b, c, d}(x)$, which provides a generalization of Theorem 15 in [6].

Theorem 1. Let $x=\left(x_{n}\right)_{n \in \mathbb{N}}$ and let $y=\mathscr{Y}_{a, b, c, d}(x)$, that is,

$$
y_{n}=\sum_{k=1}^{n} \frac{1}{n !}\left[\prod_{j=1}^{k-1}(a n+b k+c j+d)\right] B_{n, k}\left(1 ! x_{1}, 2 ! x_{2}, \ldots\right) \text { for } n \geq 1 .
$$

Assume $c \neq 0$. Then, for every $n \in \mathbb{N}$ and for any $\lambda \in \mathbb{C}$, we have

$$
\sum_{k=1}^{n}\left[\prod_{j=1}^{k-1}(\lambda-d j+d)\right] B_{n, k}(! y)=\sum_{k=1}^{n}\left[\prod_{j=1}^{k-1}(a n+b k+c j+d+\lambda)\right] B_{n, k}(! x),
$$

where $! x=\left(1 ! x_{1}, 2 ! x_{2}, \ldots\right)$ and $! y=\left(1 ! y_{1}, 2 ! y_{2}, \ldots\right)$.

Proof. Since $c \neq 0$, we can write $y_{n}$ as

$$
\begin{aligned}
y_{n} & =\sum_{k=1}^{n} \frac{1}{n !}\left[\prod_{j=1}^{k-1}(a n+b k+c j+d)\right] B_{n, k}(! x) \\
& =\sum_{k=1}^{n} \frac{1}{n !}\left[\prod_{j=1}^{k-1}(a n+(b+c) k-c j+d)\right] B_{n, k}(! x) \\
& =\sum_{k=1}^{n} \frac{c^{k-1}}{n !}\left(\begin{array}{c}
\frac{a}{c} n+\frac{(b+c)}{c} k+\frac{d}{c}-1 \\
k-1
\end{array}\right)(k-1) ! B_{n, k}(! x) .
\end{aligned}
$$

By the homogeneity property $B_{n, k}\left(c z_{1}, c z_{2}, \ldots\right)=c^{k} B_{n, k}\left(z_{1}, z_{2}, \ldots\right)$, we then conclude

$$
n ! c y_{n}=\sum_{k=1}^{n}\left(\begin{array}{c}
\frac{a}{c} n+\frac{(b+c)}{c} k+\frac{d}{c}-1 \\
k-1
\end{array}\right)(k-1) ! B_{n, k}\left(1 ! c x_{1}, 2 ! c x_{2}, \ldots\right) .
$$


If $d \neq 0$, we use Proposition 20 with $x$ replaced by $\left(1 ! c x_{1}, 2 ! c x_{2}, \ldots\right), y$ replaced by $\left(1 ! c y_{1}, 2 ! c y_{2}, \ldots\right), \alpha=a / c, \beta=(b+c) / c, \gamma=d / c$, and $\lambda$ replaced by $\lambda / c$, to obtain

$$
\begin{aligned}
\sum_{k=1}^{n}\left(\frac{d}{c}\right)^{k-1} & \left(\begin{array}{c}
\lambda / d \\
k-1
\end{array}\right)(k-1) ! B_{n, k}\left(1 ! c y_{1}, 2 ! c y_{2}, \ldots\right) \\
& =\sum_{k=1}^{n}\left(\begin{array}{c}
\frac{a}{c} n+\frac{b+c}{c} k+\frac{d}{c}-1+\frac{\lambda}{c} \\
k-1
\end{array}\right)(k-1) ! B_{n, k}\left(1 ! c x_{1}, 2 ! c x_{2}, \ldots\right) \\
& =\sum_{k=1}^{n} c\left[\prod_{j=1}^{k-1}(a n+b k+c j+d+\lambda)\right] B_{n, k}\left(1 ! x_{1}, 2 ! x_{2}, \ldots\right) .
\end{aligned}
$$

This implies

$$
\sum_{k=1}^{n} d^{k-1}\left(\begin{array}{c}
\lambda / d \\
k-1
\end{array}\right)(k-1) ! B_{n, k}(! y)=\sum_{k=1}^{n}\left[\prod_{j=1}^{k-1}(a n+b k+c j+d+\lambda)\right] B_{n, k}(! x),
$$

which gives (44) noting that $d^{k-1}\left(\begin{array}{c}\lambda / d \\ k-1\end{array}\right)(k-1) !=\prod_{j=1}^{k-1}(\lambda-d j+d)$.

If $d=0$, the above expression for $n ! c y_{n}$ reduces to

$$
n ! c y_{n}=\sum_{k=1}^{n}\left(\begin{array}{c}
\frac{a}{c} n+\frac{(b+c)}{c} k-1 \\
k-1
\end{array}\right)(k-1) ! B_{n, k}\left(1 ! c x_{1}, 2 ! c x_{2}, \ldots\right) .
$$

Using now Proposition 19 with $x$ replaced by $\left(1 ! c x_{1}, 2 ! c x_{2}, \ldots\right), y$ replaced by $\left(1 ! c y_{1}, 2 ! c y_{2}, \ldots\right)$, $\alpha=a / c, \beta=(b+c) / c$, and $\lambda$ replaced by $\lambda / c$, we get

$$
\begin{aligned}
\sum_{k=1}^{n}\left(\frac{\lambda}{c}\right)^{k-1} & B_{n, k}\left(1 ! c y_{1}, 2 ! c y_{2}, \ldots\right) \\
& =\sum_{k=1}^{n}\left(\frac{\frac{a}{c} n+\frac{b+c}{c} k-1+\frac{\lambda}{c}}{k-1}\right)(k-1) ! B_{n, k}\left(1 ! c x_{1}, 2 ! c x_{2}, \ldots\right) \\
& =\sum_{k=1}^{n} c\left[\prod_{j=1}^{k-1}(a n+b k+c j+\lambda)\right] B_{n, k}\left(1 ! x_{1}, 2 ! x_{2}, \ldots\right) .
\end{aligned}
$$

This implies

$$
\sum_{k=1}^{n} \lambda^{k-1} B_{n, k}(! y)=\sum_{k=1}^{n}\left[\prod_{j=1}^{k-1}(a n+b k+c j+\lambda)\right] B_{n, k}(! x),
$$

which is the statement of the theorem for $d=0$.

As a consequence, we obtain the following inverse relations.

Corollary 2. Let $x=\left(x_{n}\right)_{n \in \mathbb{N}}$ and $y=\mathscr{Y}_{a, b, c, d}(x)$, that is,

$$
y_{n}=\sum_{k=1}^{n} \frac{1}{n !}\left[\prod_{j=1}^{k-1}(a n+b k+c j+d)\right] B_{n, k}\left(1 ! x_{1}, 2 ! x_{2}, \ldots\right) \text { for } n \geq 1 \text {. }
$$


Further, define the polynomial $q_{n, k}(t)=t \prod_{j=1}^{k-1}(a n+d j+t)$.

(i) If $c \neq 0$, then

$$
x_{n}=\sum_{k=1}^{n} \frac{(-1)^{k-1}}{n !}\left[\frac{q_{n, k}(b+c)-q_{n, k}(b)}{c}\right] B_{n, k}\left(1 ! y_{1}, 2 ! y_{2}, \ldots\right) .
$$

In other words,

$$
\mathscr{Y}_{a, b, c, d}^{-1}=\frac{b+c}{c} \mathscr{Y}_{-a, 0,-d,-b-c}-\frac{b}{c} \mathscr{Y}_{-a, 0,-d,-b} .
$$

In particular, $\mathscr{Y}_{a, 0, c, d}^{-1}=\mathscr{Y}_{-a, 0,-d,-c}$ and $\mathscr{Y}_{a,-c, c, d}^{-1}=\mathscr{Y}_{-a, 0,-d, c}$.

(ii) If $c=0$, then

$$
x_{n}=\sum_{k=1}^{n} \frac{(-1)^{k-1}}{n !} q_{n, k}^{\prime}(b) B_{n, k}\left(1 ! y_{1}, 2 ! y_{2}, \ldots\right),
$$

where $q_{n, k}^{\prime}=\frac{d}{d t} q_{n, k}$. In particular, $\mathscr{Y}_{a, 0,0, d}^{-1}=\mathscr{Y}_{-a, 0,-d, 0}$.

Proof. First observe that $(i i)$ follows from $(i)$ by taking the limit as $c \rightarrow 0$.

If $c \neq 0$, we can apply Theorem 1 with $\lambda=-(a n+b+c+d)$ to conclude

$$
\begin{aligned}
\sum_{k=1}^{n} \frac{(-1)^{k-1}}{n !} \frac{q_{n, k}(b+c)}{c} B_{n, k}(! y) \\
=\sum_{k=1}^{n} \frac{b+c}{c n !}\left[\prod_{j=1}^{k-1}(-a n-b-c-d-d j+d)\right] B_{n, k}(! y) \\
=\sum_{k=1}^{n} \frac{b+c}{c n !}\left[\prod_{j=1}^{k-1}(b(k-1)+c(j-1))\right] B_{n, k}(! x) .
\end{aligned}
$$

Similarly, but with $\lambda=-(a n+b+d)$, we obtain

$$
\sum_{k=1}^{n} \frac{(-1)^{k-1}}{n !} \frac{q_{n, k}(b)}{c} B_{n, k}(! y)=\sum_{k=1}^{n} \frac{b}{c n !}\left[\prod_{j=1}^{k-1}(b(k-1)+c j)\right] B_{n, k}(! x) .
$$

Finally, for $k \geq 2$ we have

$$
\frac{b+c}{c} \prod_{j=1}^{k-1}(b(k-1)+c(j-1))-\frac{b}{c} \prod_{j=1}^{k-1}(b(k-1)+c j)=0,
$$

and therefore,

$$
\begin{aligned}
\sum_{k=1}^{n} \frac{(-1)^{k-1}}{n !} & {\left[\frac{q_{n, k}(b+c)-q_{n, k}(b)}{c}\right] B_{n, k}(! y) } \\
& =\frac{b+c}{c n !} B_{n, 1}(! x)-\frac{b}{c n !} B_{n, 1}(! x)=\frac{1}{n !} B_{n, 1}(! x)=x_{n} .
\end{aligned}
$$

This finishes the proof of $(i)$. 


\section{Generating Functions}

In this section, we will discuss functional equations satisfied by the generating functions of the BELL transforms $\mathscr{Y}_{a, b, c, d}$. Our approach to prove these identities showcases some of the interesting properties of partial Bell polynomials.

As before, we adopt the convenient notation $! z=\left(1 ! z_{1}, 2 ! z_{2}, \ldots\right)$.

Theorem 3. Let $x=\left(x_{n}\right)_{n \in \mathbb{N}}$ and $y=\left(y_{n}\right)_{n \in \mathbb{N}}$ be sequences such that $y=\mathscr{Y}_{a, b, c, 1}(x)$ with $c \neq 0$. Let $X(t)=\sum_{n=1}^{\infty} x_{n} t^{n}$ and $Y(t)=\sum_{n=1}^{\infty} y_{n} t^{n}$. Then

$$
X\left(t(1+Y(t))^{a}\right)=\frac{1}{c}\left[1-(1+Y(t))^{-c}\right](1+Y(t))^{-b}
$$

Proof. Let $Z(t)=1+Y(t)$. Then the right-hand side of (5) becomes

$$
\frac{1}{c}\left[1-Z(t)^{-c}\right] Z(t)^{-b}=\frac{1}{c}\left[Z(t)^{-b}-Z(t)^{-b-c}\right] .
$$

By Lemma 17, we have

$$
\begin{aligned}
n !\left[t^{n}\right] Z(t)^{-b} & =-b \sum_{k=1}^{n}\left[\prod_{j=1}^{k-1}(-b-j)\right] B_{n, k}(! y), \\
n !\left[t^{n}\right] Z(t)^{-b-c} & =-(b+c) \sum_{k=1}^{n}\left[\prod_{j=1}^{k-1}(-b-c-j)\right] B_{n, k}(! y) .
\end{aligned}
$$

Applying (44) with $d=1$ and $\lambda=-b-1$, we then get

$$
n !\left[t^{n}\right] Z(t)^{-b}=-b \sum_{k=1}^{n}\left[\prod_{j=1}^{k-1}(a n+b k+c j-b)\right] B_{n, k}(! x),
$$

and similarly, with $d=1$ and $\lambda=-b-c-1$,

$$
n !\left[t^{n}\right] Z(t)^{-b-c}=-(b+c) \sum_{k=1}^{n}\left[\prod_{j=1}^{k-1}(a n+b k+c(j-1)-b)\right] B_{n, k}(! x) .
$$

Now, for $k \geq 2$ we have

$$
\begin{aligned}
-\frac{b}{c} \prod_{j=1}^{k-1}(a n+b k & +c j-b)+\frac{b+c}{c} \prod_{j=1}^{k-1}(a n+b k+c(j-1)-b) \\
& =-\frac{b}{c} \prod_{j=1}^{k-1}(a n+b k+c j-b)+\frac{b+c}{c} \prod_{j=0}^{k-2}(a n+b k+c j-b) \\
& =a n \prod_{j=1}^{k-2}(a n+b k+c j-b)=a n \prod_{j=1}^{k-2}(a n+b(k-1)+c j),
\end{aligned}
$$


and therefore,

$$
\begin{aligned}
n !\left[t^{n}\right] \frac{1}{c}\left(Z(t)^{-b}\right. & \left.-Z(t)^{-b-c}\right) \\
& =n ! x_{n}+\sum_{k=2}^{n} a n\left[\prod_{j=1}^{k-2}(a n+b(k-1)+c j)\right] B_{n, k}(! x) \\
& =n ! x_{n}+a n \sum_{k=1}^{n-1}\left[\prod_{j=1}^{k-1}(a n+b k+c j)\right] B_{n, k+1}(! x) .
\end{aligned}
$$

Using the identity $B_{n, k+1}\left(z_{1}, z_{2}, \ldots\right)=\sum_{\ell=k}^{n-1}\left(\begin{array}{c}n-1 \\ \ell\end{array}\right) z_{n-\ell} B_{\ell, k}\left(z_{1}, z_{2}, \ldots\right)$, we get

$$
\begin{aligned}
a n \sum_{k=1}^{n-1}\left[\prod_{j=1}^{k-1}(a n+b k+c j)\right] B_{n, k+1}(! x) \\
=a \sum_{k=1}^{n-1}\left[\prod_{j=1}^{k-1}(a n+b k+c j)\right] \sum_{\ell=k}^{n-1} \frac{n !(n-\ell)}{\ell !} x_{n-\ell} B_{\ell, k}(! x) \\
=a \sum_{\ell=1}^{n-1} \frac{n !(n-\ell)}{\ell !} x_{n-\ell} \sum_{k=1}^{\ell}\left[\prod_{j=1}^{k-1}(a n+b k+c j)\right] B_{\ell, k}(! x),
\end{aligned}
$$

which implies

$$
\left[t^{n}\right] \frac{1}{c}\left(Z(t)^{-b}-Z(t)^{-b-c}\right)=x_{n}+a \sum_{\ell=1}^{n-1} \frac{n-\ell}{\ell !} x_{n-\ell} \sum_{k=1}^{\ell}\left[\prod_{j=1}^{k-1}(a n+b k+c j)\right] B_{\ell, k}(! x) .
$$

Let us next examine the left-hand side of (5). First note that if $a=0$, then (6) gives the claimed statement. If $a \neq 0$, we then use Lemma 17 and Identity (4) with $d=1$ and $\lambda=a-1$ to conclude

$$
n !\left[t^{n}\right] Z(t)^{a}=a \sum_{k=1}^{n}\left[\prod_{j=1}^{k-1}(a n+b k+c j+a)\right] B_{n, k}(! x) .
$$

Now, if $\left(w_{n}\right)_{n \in \mathbb{N}}$ is the sequence defined by $t Z(t)^{a}=\sum_{n=1}^{\infty} w_{n} t^{n}$, then $w_{1}=1$ and

$$
\frac{\ell ! w_{\ell+1}}{a}=\sum_{k=1}^{\ell}\left[\prod_{j=1}^{k-1}(a \ell+b k+c j+a)\right] B_{\ell, k}(! x) \text { for } \ell \geq 1 \text {. }
$$

Observe that, by means of (4) with $d=a$ and $\lambda=a n-a \ell-a$, we have

$$
\sum_{k=1}^{\ell}\left[\prod_{j=1}^{k-1}(a n-a \ell-a j)\right] B_{\ell, k}\left(\frac{1 ! w_{2}}{a}, \frac{2 ! w_{3}}{a}, \ldots\right)=\sum_{k=1}^{\ell}\left[\prod_{j=1}^{k-1}(a n+b k+c j)\right] B_{\ell, k}(! x),
$$

which implies

$$
\sum_{k=1}^{\ell}\left[\prod_{j=1}^{k-1}(n-\ell-j)\right] B_{\ell, k}\left(1 ! w_{2}, 2 ! w_{3}, \ldots\right)=a \sum_{k=1}^{\ell}\left[\prod_{j=1}^{k-1}(a n+b k+c j)\right] B_{\ell, k}(! x) .
$$


On the other hand, Faà di Bruno's formula combined with Equation $(3 \ell)$ in [18, Sec. 3.3] gives

$$
\begin{aligned}
n ![t]^{n} X\left(t Z(t)^{a}\right) & =\sum_{\ell=1}^{n} \ell ! x_{\ell} B_{n, \ell}\left(1 ! w_{1}, 2 ! w_{2}, \ldots\right) \\
& =\sum_{\ell=1}^{n} \ell ! x_{\ell} \sum_{k=2 \ell-n}^{\ell} \frac{n !}{(n-\ell) ! k !} B_{n-\ell, \ell-k}\left(1 ! w_{2}, 2 ! w_{3}, \ldots\right) \\
& =\sum_{\ell=1}^{n} \ell ! x_{\ell} \sum_{k=0}^{n-\ell} \frac{n !}{(n-\ell) !(\ell-k) !} B_{n-\ell, k}\left(1 ! w_{2}, 2 ! w_{3}, \ldots\right) \\
& =\sum_{\ell=0}^{n-1}(n-\ell) ! x_{n-\ell} \sum_{k=0}^{\ell} \frac{n !}{\ell !(n-\ell-k) !} B_{\ell, k}\left(1 ! w_{2}, 2 ! w_{3}, \ldots\right) .
\end{aligned}
$$

Since $\ell=0$ gives $n ! x_{n}$, and since $\frac{(n-\ell) !}{(n-\ell-k) !}=(n-\ell) \prod_{j=1}^{k-1}(n-\ell-j)$, we arrive at

$$
\left[t^{n}\right] X\left(t Z(t)^{a}\right)=x_{n}+\sum_{\ell=1}^{n-1} \frac{n-\ell}{\ell !} x_{n-\ell} \sum_{k=1}^{n-\ell}\left[\prod_{j=1}^{k-1}(n-\ell-j)\right] B_{\ell, k}\left(1 ! w_{2}, 2 ! w_{3}, \ldots\right) .
$$

At last, the theorem follows by combining (6), (7), and (8)).

Corollary 4. Let $x=\left(x_{n}\right)_{n \in \mathbb{N}}$ and $y=\left(y_{n}\right)_{n \in \mathbb{N}}$ be sequences such that $y=\mathscr{Y}_{a, b, c, d}(x)$. Let $X(t)=\sum_{n=1}^{\infty} x_{n} t^{n}$ and $Y(t)=\sum_{n=1}^{\infty} y_{n} t^{n}$. Then:

(i) If $c \neq 0$ and $d \neq 0$,

$$
X\left(t(1+d Y(t))^{a / d}\right)=\frac{1}{c}\left[1-(1+d Y(t))^{-c / d}\right](1+d Y(t))^{-b / d} .
$$

(ii) If $c=0$ and $d \neq 0$,

$$
X\left(t(1+d Y(t))^{a / d}\right)=\log \left((1+d Y(t))^{1 / d}\right)(1+d Y(t))^{-b / d} .
$$

(iii) If $c \neq 0$ and $d=0$,

$$
X\left(t e^{a Y(t)}\right)=\frac{1}{c}\left[1-e^{-c Y(t)}\right] e^{-b Y(t)} .
$$

(iv) If $c=d=0$,

$$
X\left(t e^{a Y(t)}\right)=Y(t) e^{-b Y(t)} .
$$

Proof. Assume $d \neq 0$. If we let $\hat{x}=\left(d x_{n}\right)_{n \in \mathbb{N}}$ and $\hat{y}=\mathscr{Y}_{\frac{a}{d}, \frac{b}{d}, \frac{c}{d}, 1}(\hat{x})$, then (15) implies

$$
\widehat{X}\left(t(1+\widehat{Y}(t))^{a / d}\right)=\frac{d}{c}\left[1-(1+\widehat{Y}(t))^{-c / d}\right](1+\widehat{Y}(t))^{-b / d} .
$$

Using (11) together with the homogeneity of the partial Bell polynomials, it is easy to check that $\widehat{Y}(t)=d Y(t)$. Since $\widehat{X}(t)=d X(t)$, the above identity gives $(i)$. 
The claimed identity in ( $i i)$ follows from the identity in $(i)$ by taking the limit as $c \rightarrow 0$, and (iii) follows from $(i)$ by taking the limit as $d \rightarrow 0$. The statement in $(i v)$ follows from any of the previous items with a corresponding limit argument.

\section{A convolution formula}

In this section we consider a class of sequences, defined through BELL transforms, that is amenable to convolutions of arbitrary length.

Once again, we use the notation $! z=\left(1 ! z_{1}, 2 ! z_{2}, \ldots\right)$.

Proposition 5. Let $x=\left(x_{n}\right)_{n \in \mathbb{N}}$ and let $y=\mathscr{Y}_{a, b, c, d}(x)$ with $d \neq 0$. Let $\hat{y}=d \cdot \mathscr{Y}_{a, b, c, d}(x)$, that is, $\left(\hat{y}_{n}\right)_{n \in \mathbb{N}}$ is the sequence defined by

$$
\hat{y}_{n}=d \cdot \sum_{k=1}^{n} \frac{1}{n !}\left[\prod_{j=1}^{k-1}(a n+b k+c j+d)\right] B_{n, k}(! x) \text { for } n \geq 1 .
$$

If we let $\hat{y}_{0}=1$ and $r \in \mathbb{N}$, we have

$$
\sum_{m_{1}+\cdots+m_{r}=n} \hat{y}_{m_{1}} \cdots \hat{y}_{m_{r}}=d r \cdot \sum_{k=1}^{n} \frac{1}{n !}\left[\prod_{j=1}^{k-1}(a n+b k+c j+d r)\right] B_{n, k}(! x) .
$$

Proof. First note that if $Y(t)=\sum_{n=1}^{\infty} y_{n} t^{n}$, then the expression $\sum \hat{y}_{m_{1}} \cdots \hat{y}_{m_{r}}$ is given by the coefficients of $(1+d Y(t))^{r}$. By Lemma 17, we have

$$
\begin{aligned}
n !\left[t^{n}\right](1+d Y(t))^{r} & =r \sum_{k=1}^{n}\left[\prod_{j=1}^{k-1}(r-j)\right] B_{n, k}(!(d y)) \\
& =r \sum_{k=1}^{n} d^{k}\left[\prod_{j=1}^{k-1}(r-j)\right] B_{n, k}(! y) \\
& =d r \sum_{k=1}^{n}\left[\prod_{j=1}^{k-1}(d r-d j)\right] B_{n, k}(! y)
\end{aligned}
$$

Applying (44) with $\lambda=d r-d$, we get

$$
\sum_{k=1}^{n}\left[\prod_{j=1}^{k-1}(d r-d j)\right] B_{n, k}(! y)=\sum_{k=1}^{n}\left[\prod_{j=1}^{k-1}(a n+b k+c j+d r)\right] B_{n, k}(! x),
$$

which gives the claimed convolution formula.

Remark. If $d=1$, we have $\hat{y}=y=\mathscr{Y}_{a, b, c, 1}(x)$. If in addition $c=-1$, we may write the sequence $\left(y_{n}\right)_{n \in \mathbb{N}}$ as

$$
y_{n}=\sum_{k=1}^{n}\left(\begin{array}{c}
a n+b k \\
k-1
\end{array}\right) \frac{(k-1) !}{n !} B_{n, k}(! x)
$$


and the above convolution formula (with $y_{0}=1$ ) becomes

$$
\sum_{m_{1}+\cdots+m_{r}=n} y_{m_{1}} \cdots y_{m_{r}}=r \cdot \sum_{k=1}^{n}\left(\begin{array}{c}
a n+b k+r-1 \\
k-1
\end{array}\right) \frac{(k-1) !}{n !} B_{n, k}(! x) .
$$

As discussed by the authors in [7], this formula may be used (with appropriate choices of the parameters $a$ and $b$ ) to obtain convolution formulas of known sequences like Fibonacci, Jacobsthal, Motzkin, Fuss-Catalan, and many others.

Corollary 6. For $a, b \in \mathbb{R}$, not both equal to 0 , the sequence $\left(y_{n}\right)_{n \in \mathbb{N}}$ defined by (9) satisfies the recurrence

$$
y_{n}=\sum_{\ell=1}^{n} x_{\ell} \sum_{i_{1}+\cdots+i_{a \ell+b}=n-\ell} y_{i_{1}} \ldots y_{i_{a \ell+b}}
$$

where each $i_{j}$ is a nonnegative integer and $y_{0}=1$.

A detailed proof of (10) can be found in [5, Prop. 3.3].

\section{EXAMPLES}

Example 7 (IDENTITY). If $a=b=c=d=0$, we get $y_{n}=\frac{1}{n !} B_{n, 1}(! x)=x_{n}$ for every $n \in \mathbb{N}$. Thus $\mathscr{Y}_{0,0,0,0}$ is the identity map.

Example 8 (EXP). If $a=b=c=0$ and $d=1$, then

$$
X(t)=\log (1+Y(t)) \text {, or equivalently, } 1+Y(t)=e^{X(t)} .
$$

Therefore, a sequence $\left(b_{n}\right)$ is the EXP transform of $\left(a_{n}\right)$, as defined in [4], if and only if $y=\left(b_{n} / n !\right)$ is the BeLL transform of $x=\left(a_{n} / n !\right)$ with parameters $(0,0,0,1)$.

Example 9 (INVERT). If $a=b=0$ and $c=d=m$, then

$$
X(t)=\frac{1}{m}\left[1-(1+m Y(t))^{-1}\right], \text { or equivalently, } 1+m Y(t)=\frac{1}{1-m X(t)} .
$$

For $m \in \mathbb{N}, \mathscr{Y}_{0,0, m, m}(x)$ is the $m$-th INVERT transform of $x$.

Example $10(\mathrm{CONV})$. If $a=b=0, c=-1$, and $d=m$, then

$$
X(t)=-1+(1+m Y(t))^{1 / m}, \text { which implies }(1+X(t))^{m}=1+m Y(t) .
$$

In particular, if $m=2$ and $\left(x_{n}\right)$ is such that $x_{0}=1$, then $\mathscr{Y}_{0,0,-1,2}=\frac{1}{2} \mathrm{CONV}$.

Example 11 (REVERT). The REVERT transform, as it was defined in 4, is closely related to series reversion. Given a sequence $\left(a_{n}\right)$ with $a_{1}=1$, let $A(t)=\sum_{n=1}^{\infty} a_{n} t^{n}$. The REvERT transform of $\left(a_{n}\right)$ is the sequence $\left(b_{n}\right)$ such that $B(t)=\sum_{n=1}^{\infty}(-1)^{n+1} b_{n} t^{n}$ is the power series inverse of $A(t)$. Thus $B(A(t))=t$ and $b_{1}=1$.

In order to express REVERT in terms of a BELL transform, we first recall the following fact (see e.g. [15, Corollary 11.3]):

$$
\text { if } \phi(t)=t\left(1+\sum_{r=1}^{\infty} \alpha_{r} \frac{t^{r}}{r !}\right) \text {, then } \phi^{-1}(u)=u\left(1+\sum_{n=1}^{\infty} \beta_{n} \frac{u^{n}}{n !}\right) \text {, }
$$


where

$$
\beta_{n}=\sum_{k=1}^{n}(-1)^{k} \frac{(n+k) !}{(n+1) !} B_{n, k}\left(\alpha_{1}, \alpha_{2}, \ldots\right) .
$$

Observe that, since $a_{1}=1$, we have $A(t)=t\left(1+\sum_{n=1}^{\infty} a_{n+1} t^{n}\right)$. So, if we let $\alpha_{r}=r ! a_{r+1}$, the above inversion formula gives

$$
n !(-1)^{n} b_{n+1}=\sum_{k=1}^{n}(-1)^{k} \frac{(n+k) !}{(n+1) !} B_{n, k}\left(1 ! a_{2}, 2 ! a_{3}, \ldots\right) .
$$

In other words, for $n \geq 1$,

$$
b_{n+1}=\sum_{k=1}^{n} \frac{1}{n !}\left[\prod_{j=1}^{k-1}(-n-j-1)\right] B_{n, k}\left(1 ! a_{2},-2 ! a_{3}, 3 ! a_{4},-4 ! a_{5}, \ldots\right),
$$

which is a BeLL transform with $a=-1, b=0, c=d=-1$. Using the operators

$$
\begin{aligned}
L \circ\left(x_{1}, x_{2}, \ldots\right) & =\left(x_{2}, x_{3}, \ldots\right), \\
R \circ\left(x_{1}, x_{2}, \ldots\right) & =\left(1, x_{1}, x_{2}, \ldots\right), \\
I \circ\left(x_{1}, x_{2}, \ldots\right) & =\left(x_{1},-x_{2}, x_{3},-x_{4}, \ldots\right),
\end{aligned}
$$

we get

$$
\text { REVERT }=R \circ \mathscr{Y}_{-1,0,-1,-1} \circ I \circ L .
$$

Finally, if $X(t)=\sum_{n=1}^{\infty}(-1)^{n} a_{n+1} t^{n}$ and $Y(t)=\sum_{n=1}^{\infty} b_{n+1} t^{n}$, then

$$
X(t(1-Y(t)))=-1+(1-Y(t))^{-1}=\frac{Y(t)}{1-Y(t)} .
$$

Example 12 (polygon Dissection). If $a=1, b=0, c=d=1$, then

$$
X(t(1+Y(t)))=1-(1+Y(t))^{-1}=\frac{Y(t)}{1+Y(t)} .
$$

This transformation, which is clearly related to REVERT, plays a role in the enumeration of polygon dissections by noncrossing diagonals. In particular, if $\mathbb{1}=(1,1,1, \ldots)$ is the sequence of ones, then $\mathscr{Y}_{1,0,1,1}(\mathbb{1})$ gives the little Schröder numbers 1, 1, 3, 11, 45, 197, 903, 4279, 20793, ... cf. [34, A001003]. A comprehensive discussion on polygon dissections can be found in [8].

Example 13 (nOnCrossing PARtition). If $a=1, b=0, c=-1, d=1$, then

$$
X(t(1+Y(t)))=-[1-(1+Y(t))]=Y(t) .
$$

This is the functional equation for the NONCROSSING PARTITION transform as defined by Callan in [13, Eqn. (4)]. As already mentioned in the introduction, $\mathscr{Y}_{1,0,-1,1}(\mathbb{1})$ gives the sequence of Catalan numbers.

Remark. Observe that, since $\mathscr{Y}_{a, b, c, d}=\mathscr{Y}_{a, b+c,-c, d}$, the INVERT transform is also given by $\mathscr{Y}_{0,1,-1,1}$, and the POLYGON DISSECTION transform is given by $\mathscr{Y}_{1,1,-1,1}$. In other words, the transformations discussed in Examples 9, 12, and 13, are all special instances of the BELL 
transform $\mathscr{Y}_{a, b,-1,1}$. In this case, the elements of the sequence $\mathscr{Y}_{a, b,-1,1}(x)$ may be written as in (9), and the generating functions of $\left(x_{n}\right)_{n \in \mathbb{N}}$ and $\left(y_{n}\right)_{n \in \mathbb{N}}$ satisfy

$$
X\left(t(1+Y(t))^{a}\right)=Y(t)(1+Y(t))^{-b} .
$$

This particular family of transforms plays a role in the enumeration of colored Dyck paths, colored dissections of convex polygons, colored rooted trees, planar maps, permutations, and more. Some of these applications will be discussed in the next section.

We end this section with a brief discussion of the family $\mathscr{T}_{m} \stackrel{\text { def }}{=} \mathscr{Y}_{m, 0,-1,1}$.

Example 14. For $x=\left(x_{n}\right)_{n \in \mathbb{N}}$ and $m \in \mathbb{Z}$, the sequence $y=\mathscr{T}_{m}(x)$ may be written as

$$
y_{n}=\sum_{k=1}^{n}\left(\begin{array}{c}
m n \\
k-1
\end{array}\right) \frac{(k-1) !}{n !} B_{n, k}\left(1 ! x_{1}, 2 ! x_{2}, \ldots\right),
$$

and in terms of generating functions, we have

$$
X\left(t(1+Y(t))^{m}\right)=Y(t)
$$

In particular, if $x=(1,1, \ldots)$, then

$$
\begin{aligned}
y_{n} & =\sum_{k=1}^{n}\left(\begin{array}{c}
m n \\
k-1
\end{array}\right) \frac{(k-1) !}{n !} B_{n, k}(1 !, 2 !, \ldots) \\
& =\sum_{k=1}^{n} \frac{1}{k}\left(\begin{array}{c}
m n \\
k-1
\end{array}\right)\left(\begin{array}{c}
n-1 \\
k-1
\end{array}\right) \\
& =\sum_{k=1}^{n} \frac{1}{m n+1}\left(\begin{array}{c}
m n+1 \\
k
\end{array}\right)\left(\begin{array}{c}
n-1 \\
n-k
\end{array}\right)=\frac{1}{m n+1}\left(\begin{array}{c}
(m+1) n \\
n
\end{array}\right) .
\end{aligned}
$$

Thus $\mathscr{T}_{m}(\mathbb{1})$ gives the Fuss-Catalan numbers (see e.g. [1, 25, 33]), which for $m \in \mathbb{N}$ enumerate the $m$-divisible noncrossing partitions of $[m n]$, cf. [2, 21].

In general, $\mathscr{T}_{0}$ is the identity map, and by Lemma 18 with $\alpha=m, \beta=0$, and $\lambda=m^{\prime} \cdot n$, we obtain

$$
\mathscr{T}_{m^{\prime}} \circ \mathscr{T}_{m}=\mathscr{T}_{m+m^{\prime}} \text {, and consequently, } \mathscr{T}_{m}^{-1}=\mathscr{T}_{-m}
$$

This shows that, for $m \in \mathbb{N}, \mathscr{T}_{m}(x)$ is the $m$-th NONCROSSING PARTITION transform of $x$.

Here is a connection between the NONCROSSING PARTITION transform and the BINOMIAL transform, as defined in [4].

Example 15. Let $\mathcal{S}_{\nu}$ be the operator defined by

$$
\mathcal{S}_{\nu} \circ\left(x_{1}, x_{2}, x_{3}, \ldots\right)=\left(x_{1}+\nu, x_{2}, x_{3}, \ldots\right) .
$$

Given $a=\left(a_{0}, a_{1}, \ldots\right)$, let $b=\operatorname{BinOmiaL}(a)$ be the sequence defined by

$$
b_{n}=\sum_{k=0}^{n}\left(\begin{array}{l}
n \\
k
\end{array}\right) a_{k} \text { for } n \in \mathbb{N}_{0} .
$$

Then for $\nu \in \mathbb{N}$, and with $L$ and $R$ as in (12), we have

$$
\mathscr{T}_{1} \circ \mathcal{S}_{\nu}=L \circ \text { BINOMIAL }^{\nu} \circ R \circ \mathscr{T}_{1} .
$$




\section{Application: Combinatorial structures}

In this section we present a few examples that illustrate how BELL transforms may be used to link the enumeration of certain classes of combinatorial structures with the enumeration of suitable building blocks that serve as primitive elements within each class.

Rational Dyck paths. A class of objects that nicely demonstrates the above principle is the class of rational Dyck paths. Let $\mathcal{D}_{\beta / \alpha}(n)$ be the set of lattice paths from $(0,0)$ to $(\alpha n, \beta n)$ which may touch but never rise above the line $\alpha y=\beta x$, where $n, \alpha$, and $\beta$ are positive integers with $\operatorname{gcd}(\alpha, \beta)=1$. Further, let $\mathcal{D}_{\beta / \alpha}^{*}(n)$ be the set of paths in $\mathcal{D}_{\beta / \alpha}(n)$ that stay strongly below the line $\alpha y=\beta x$ (except at the endpoints). Clearly, the elements in $\mathcal{D}_{\beta / \alpha}(n)$ that are not in $\mathcal{D}_{\beta / \alpha}^{*}(n)$ can be thought of as concatenations of shorter paths from the sets $\mathcal{D}_{\beta / \alpha}^{*}(1), \mathcal{D}_{\beta / \alpha}^{*}(2), \ldots, \mathcal{D}_{\beta / \alpha}^{*}(n-1)$, so it is not surprising that the sequence $\phi_{n}=\left|\mathcal{D}_{\beta / \alpha}(n)\right|$ is the INVERT transform of $\psi_{n}=\left|\mathcal{D}_{\beta / \alpha}^{*}(n)\right|$. This fact, proved in [10], implies

$$
\phi_{n}=\sum_{k=1}^{n} \frac{k !}{n !} B_{n, k}\left(1 ! \psi_{1}, 2 ! \psi_{2}, \ldots\right) .
$$

In other words, with $\phi=\left(\phi_{n}\right)_{n \in \mathbb{N}}$ and $\psi=\left(\psi_{n}\right)_{n \in \mathbb{N}}$, we have

$$
\phi=\mathscr{Y}_{0,1,-1,1}(\psi) \text {. }
$$

However, a different interpretation is possible if we think of rational paths as words over the alphabet $\{a, b\}$. This may be done by associating the letter $a$ with the step $(1,0)$ and the letter $b$ with the step $(0,1)$. The elements of $\mathcal{D}_{\beta / \alpha}=\bigcup_{n} \mathcal{D}_{\beta / \alpha}(n)$ then correspond to a generalized Dyck language over $\{a, b\}$ having valuations $h(a)=\beta$ and $h(b)=-\alpha$. In [20] Duchon established a connection between the set $\mathcal{D}_{\beta / \alpha}$ and its subset of corresponding factor-free words 1 . In fact, if $\theta_{n}$ denotes the number of factor-free words in $\mathcal{D}_{\beta / \alpha}(n)$, then

$$
\phi_{n}=\sum_{k=1}^{n}\left(\begin{array}{c}
(\alpha+\beta) n \\
k-1
\end{array}\right) \frac{(k-1) !}{n !} B_{n, k}\left(1 ! \theta_{1}, 2 ! \theta_{2}, \ldots\right) .
$$

That is,

$$
\phi=\mathscr{Y}_{\alpha+\beta, 0,-1,1}(\theta) .
$$

As shown in [5], equation (14) implies that the elements of $\mathcal{D}_{\beta / \alpha}(n)$ are in bijection to a class of Dyck paths with ascents of length $(\alpha+\beta) j$ (for $j=1, \ldots, n$ ) that may be colored in $\theta_{j}$ different ways. As a consequence, every path in $\mathcal{D}_{\beta / \alpha}$ may be constructed from factor-free words through an insertion process determined by the corresponding $\theta$-colored Dyck paths. An explicit algorithm for this construction can be found in [9, Section 2].

Finally, we have Bizley's formula (see [10]):

$$
\phi_{n}=\sum_{k=1}^{n} \frac{1}{n !} B_{n, k}\left(1 ! f_{1}, 2 ! f_{2}, \ldots\right),
$$

where $f_{j}=\frac{1}{(\alpha+\beta) j}\left(\begin{array}{c}(\alpha+\beta) j \\ \alpha j\end{array}\right)$ for $j \in \mathbb{N}$. In other words,

$$
\phi=\mathscr{Y}_{0,0,0,1}(f),
$$

\footnotetext{
${ }^{1} \mathrm{~A}$ word in a language $L$ is said to be factor-free if it has no proper factor in $L$.
} 
which means that the sequence $\left(n ! \phi_{n}\right)_{n \in \mathbb{N}}$ is the EXP transform of $\left(n ! f_{n}\right)_{n \in \mathbb{N}}$. This formula reflects the enumeration of $\mathcal{D}_{\beta / \alpha}(n)$ using cyclical permutations of its elements.

Rooted planar maps. In [37] Tutte studied the enumeration of rooted planar maps and established a link to the enumeration of nonseparable rooted planar maps, which under the action of $\mathscr{Y}_{2,0,-1,1}$ may be considered their prime elements.

Let $a_{n}$ be the number of rooted planar maps with $n$ edges and let $b_{n}$ be the number of nonseparable rooted planar maps with $n$ edges. Let $A(t)$ and $B(t)$ be the generating functions for $a=\left(a_{n}\right)_{n \in \mathbb{N}}$ [34, A000168] and $b=\left(b_{n}\right)_{n \in \mathbb{N}}$ [34, A000139], respectively. As proved by Tutte (cf. [37, Equation 6.3]), these functions satisfy the functional equation

$$
A(t)=B\left(t(1+A(t))^{2}\right)
$$

which by Theorem 3 implies

$$
a=\mathscr{Y}_{2,0,-1,1}(b) .
$$

In particular, using the results from [5], we conclude:

There is a bijection between the set of rooted planar maps with $n$ edges and the set of Dyck paths of semilength $2 n$ having ascents of even length, and such that each $2 j$-ascent may be colored in $b_{j}$ different ways.

Bicubic maps. Another example amenable to BeLL transformations is given by the following connection between rooted bicubic planar maps and their subclass of 3-connected elements. In [37, Section 11] Tutte observed that "Each rooted bicubic map can be represented as a multiple extension of a 3-connected bicubic core" and proved the functional equation

$$
F(t)=G\left(t(1+F(t))^{3}\right),
$$

where $F(t)=\sum f_{n} t^{n}$ enumerates the rooted bicubic maps of $2 n$ vertices and $G(t)=\sum g_{n} t^{n}$ counts those maps that are 3-connected. This means

$$
f=\mathscr{Y}_{3,0,-1,1}(g) .
$$

Therefore, using the results from [5] once again, we conclude:

There is a bijection between the set of rooted bicubic maps of $2 n$ vertices and the set of Dyck paths of semilength $3 n$ having ascents of length multiple of 3 , and such that each $3 j$-ascent may be colored in $g_{j}$ different ways.

Note that the relation $g=\mathscr{Y}_{-3,0,-1,1}(f)$ provides the identity

$$
g_{n}=\sum_{k=1}^{n}\left(\begin{array}{c}
-3 n \\
k-1
\end{array}\right) \frac{(k-1) !}{n !} B_{n, k}\left(1 ! f_{1}, 2 ! f_{2}, \ldots\right) .
$$

Since $f_{j}=\frac{3(2 j-1) ! 2^{j}}{(j-1) !(j+2) !}$ for $j \geq 1$ (see [37] and [34, A000257]), the above formula 2 provides a way to compute $g_{n}$. Here are the first few values:

$$
1,0,0,1,0,3,7,15,63,168,561,1881,6110,21087, \ldots \text { ([34, A298358]). }
$$

\footnotetext{
${ }^{2}$ Which may be written in terms of binomials using Lemma 21
} 
Eulerian maps. It is well known that rooted bicubic planar maps of $2 n$ vertices are in one-toone correspondence with rooted Eulerian planar maps with $n$ edges. Moreover, as pointed out in [28], the functional equation (15) remains valid when restricted to the class of rooted Eulerian planar maps. Thus, if $F(t)$ is as in (16) and $H(t)=\sum h_{n} t^{n}$ is the generating function for the nonseparable rooted Eulerian maps with $n$ edges (cf. [34, A069728]), then

$$
F(t)=H\left(t(1+F(t))^{2}\right) .
$$

In other words, $f=\mathscr{Y}_{2,0,-1,1}(h)$ and we get the formula

$$
h_{n}=\sum_{k=1}^{n}\left(\begin{array}{c}
-2 n \\
k-1
\end{array}\right) \frac{(k-1) !}{n !} B_{n, k}\left(1 ! f_{1}, 2 ! f_{2}, \ldots\right) .
$$

Permutations. Let $S_{n}$ be the set of permutations on $[n]=\{1, \ldots, n\}$, and let $p$ denote the sequence $(n !)_{n \in \mathbb{N}}$. The inverse of $p$ under the INVERT and NONCROSSING PARTITION transforms,

$$
x_{\text {ind }}=\mathscr{Y}_{0,1,-1,1}^{-1}(p) \text { and } x_{\text {sif }}=\mathscr{Y}_{1,0,-1,1}^{-1}(p),
$$

enumerate two known classes of permutations that serve as building blocks for all of the elements of $S_{n}$. These are sequences A003319 $\left(x_{\text {ind }}\right)$ and A075834 $\left(x_{\text {sif }}\right)$ in [34].

Stabilized-interval-free permutations. As introduced by Callan in [12], a permutation on $[n]$ is said to be stabilized-interval-free (SIF) if it does not stabilize any proper subinterval of $[n]$. The sequence $x_{\text {sif }}$ counts the SIF permutations on [n], see [34, A075834].

The fact that $(n !)_{n \in \mathbb{N}}$ is the NONCROSSING PARTITION transform of $x_{\text {sif }}$ was proved and discussed in [12. As expected, there is a connection to Dyck paths that provides a natural way to visualize the unique decomposition of a permutation on $[n]$ into SIF permutations of length less than or equal to $n$. Specifically, (18) implies that:

The set of permutations on $[n]$ is in one-to-one correspondence with the set of Dyck paths of semilength $n$ such that each $j$-ascent may be colored in as many ways as the number of SIF permutations of length $j$.

Indecomposable permutations. The sequence $x_{\text {ind }}$ enumerates the class of indecomposable permutations as introduced by Comtet [17. This reflects the fact that every permutation on $[n]$ can be split into indecomposable permutations of length less than or equal to $n$, so they play the role of building blocks from which all permutations can be constructed. In fact, every permutation on $[n]$ may be represented as a composition of $n$ whose parts of length $j$ are labeled by indecomposable permutations of length $j$.

Using this interpretation it is easy to see that, if a pattern $\sigma$ is indecomposable, then every permutation in $\operatorname{Av}(\sigma)$ (set of permutations avoiding the pattern $\sigma$ ) can be split into $\sigma$-avoiding indecomposable permutations (denoted by $\operatorname{Av}^{\text {ind }}(\sigma)$ ). Thus if $a_{\sigma}=\left(\operatorname{Av}_{n}(\sigma)\right)_{n \in \mathbb{N}}$ and $i_{\sigma}=\left(\operatorname{Av}_{n}^{\text {ind }}(\sigma)\right)_{n \in \mathbb{N}}$, then we have (cf. [23, Lem. 3.1])

$$
i_{\sigma}=\mathscr{Y}_{0,1,-1,1}^{-1}\left(a_{\sigma}\right) \text {. }
$$

For example, since the class $\operatorname{Av}(2413)$ is enumerated by [34, A022558] (cf. [11, 35]), and since this sequence is the INVERT transform of the right-shifted [34, A000257], we conclude

$$
\operatorname{Av}_{n}^{\text {ind }}(\sigma)=f_{n-1}=\frac{3(2 n-3) ! 2^{n-1}}{(n-2) !(n+1) !} \text { for } n \geq 2,
$$


for every $\sigma \in\{2413,2431,3142,3241,4132,4213\}$. In addition, with $f_{0}=1$ we get

$$
\operatorname{Av}_{n}(\sigma)=\sum_{k=1}^{n} \frac{k !}{n !} B_{n, k}\left(1 ! f_{0}, 2 ! f_{1}, 3 ! f_{2}, \ldots\right) .
$$

Similarly, since $\operatorname{Av}(\sigma, \tau)$ for $(\sigma, \tau) \in\{(321,2341),(321,3412),(321,3142)\}$ is enumerated by [34, A001519], we can use (19) to obtain

$$
\operatorname{Av}_{n}^{\text {ind }}(\sigma, \tau)=2^{n-2} \text { for } n \geq 2 .
$$

Finally, if $(\sigma, \tau)$ is any of the following pairs of permutations:

$$
\begin{array}{lll}
(4321,4312), & (4312,4231), & (4312,4213), \\
(4312,3412), & (4231,4213), & (4213,4132), \\
(4213,4123), & (4213,2413), & (3142,2413),
\end{array}
$$

it was shown by Kremer [27] that $\operatorname{Av}_{n}(\sigma, \tau)$ is the large Schröder number A006318(n-1). Hence (19) gives that $\operatorname{Av}_{n}^{\text {ind }}(\sigma, \tau)$ equals the little Schröder number A001003( $\left.n-1\right)$.

The class $\operatorname{Av}(2413,3412)$. BELL transformations can be combined with the OEIS [34] to create flows of sequences associated with a particular sequence of interest. This often leads to combinatorial connections that can be verified through the functional equation satisfied by the generating functions.

Let us illustrate this strategy with the sequence A000257 (number of rooted bicubic maps of $2 n$ vertices, number of rooted Eulerian maps with $n$ edges, or $A v_{n+1}^{\text {ind }}(2413)$, for instance). Using BELL transformations one discovers the following apparent connections:

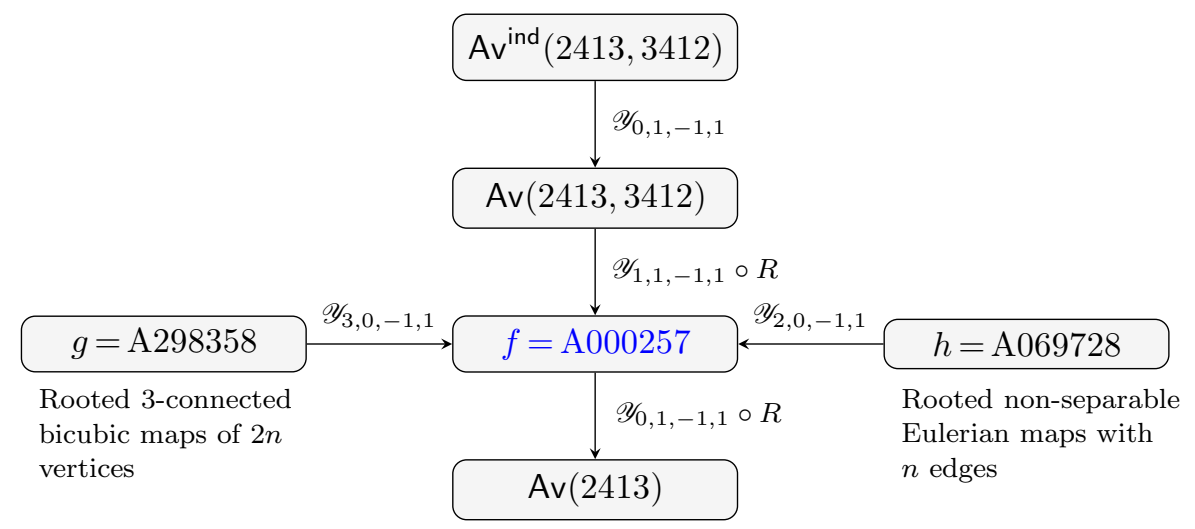

where $R$ is the right-shift operator $R \circ\left(x_{1}, x_{2}, \ldots\right)=\left(1, x_{1}, x_{2}, \ldots\right)$.

On the one hand, the identities

$$
f=\mathscr{Y}_{3,0,-1,1}(g), \quad f=\mathscr{Y}_{2,0,-1,1}(h), \text { and } \operatorname{Av}_{n}(2413)=\mathscr{Y}_{0,1,-1,1}(R(f))
$$

are indeed true and consistent with the previously discussed building block approach. On the other hand, the connection between $\operatorname{Av}(2413,3412)$ and $f$ is quite surprising. Note that, since $\mathscr{Y}_{1,1,-1,1}^{-1}=\mathscr{Y}_{-1,0,-1,-1}$, the conjectured identity is equivalent to

$$
R \circ \operatorname{Av}(2413,3412)=\mathscr{Y}_{-1,0,-1,-1}(f) .
$$


If $A(t)$ is the generating function for $\operatorname{Av}(2413,3412)$, then the right-shifted sequence has generating function $t \mathcal{A}(t)$, where $\mathcal{A}(t)=1+A(t)$. Hence (20) together with Corollary 4 gives the functional equation

$$
1+F(t(1-t \mathcal{A}(t)))=\frac{1}{1-t \mathcal{A}(t)} .
$$

Recall that $F(t)$ is the generating function for the sequence A000257. Now, using the known expression

$$
F(t)=\frac{1}{32 t^{2}}\left(-1+12 t-24 t^{2}+(1-8 t)^{3 / 2}\right),
$$

and letting $\mathcal{F}(t)=1+F(t)$, one can verify the identity

$$
16 t^{2} \mathcal{F}(t)^{2}-\left(8 t^{2}+12 t-1\right) \mathcal{F}(t)+t^{2}+11 t-1=0,
$$

which leads to the functional equation

$$
t^{4} \mathcal{A}(t)^{3}+\left(5 t^{3}-11 t^{2}\right) \mathcal{A}(t)^{2}+\left(3 t^{2}+10 t-1\right) \mathcal{A}(t)-9 t+1=0 .
$$

The validity of this equation was very recently proved by Miner and Pantone [32], thus (20) holds. As a consequence, we get the formula

$$
a_{n-1}=\sum_{k=1}^{n}\left(\begin{array}{c}
-n-2 \\
k-1
\end{array}\right) \frac{(k-1) !}{n !} B_{n, k}\left(1 ! f_{1}, 2 ! f_{2}, \ldots\right) \text { for } n \geq 2,
$$

which may be written in terms of binomials using Lemma 21 .

\section{Concluding Remarks}

The goal of this paper is to introduce a family of BELL transformations and to discuss their main properties and combinatorial interpretations. We discussed several instances of these transformations and showcased their unifying principle. The predominant feature has been the use of partial Bell polynomials. As we have shown here, they provide a suitable machinery for dealing with problems that involve Lagrange inversion and to problems that lead to recurrence relations of convolution type.

We have also shown that, given a class of irreducible/primitive combinatorial objects (building blocks), BELL transformations offer an efficient tool for the enumeration of "composite" objects constructed from the given set of building blocks. This principle has already been illustrated in Section 6, but it is only appropriate to add a brief discussion about the Bell numbers $\left(B_{n}\right)_{n \in \mathbb{N}}$ (see [34, A000110]). It is well-known that $B_{n}$ gives the number of set partitions of $[n]$. They can be built from connected partitions (enumerated by A099947 in [34]) through a standard noncrossing insertion procedure, and if $b$ denotes the sequence $\left(B_{n}\right)_{n \in \mathbb{N}}$, then $b=\mathscr{Y}_{1,0,-1,1}(A 099947)$. Alternatively, set partitions can also be built from irreducible partitions (enumerated by A074664 in [34]) through concatenation and shifting, and we have $b=\mathscr{Y}_{0,1,-1,1}(A 074664)$. Finally, there is the natural connection via the EXP transform: $\left(n ! B_{n}\right)=\mathscr{Y}_{0,0,0,1}(x)$, where $x_{n}=\frac{1}{n !}$.

Such connections via BELL transformations can be found effortlessly, and very often they provide a source of combinatorial insight. In addition, the representation in terms of partial Bell polynomials allows for efficient counting, in particular if one is interested in restricting the building blocks used for the construction of the combinatorial objects at hand. 
While the applications we considered in this paper are purely combinatorial, it is worth pointing out that partial Bell polynomials and functional equations like the ones in Section 3 are certainly relevant in other areas of research, most recently in quantum field theory (see e.g. [24, 26]). The few applications we chose to discuss in Section 66 are entirely based on our current interests and are by no means a sign of limitations. We hope that our results serve as motivation to explore other aspects of the BELL transformations. Algebraically, it would be interesting to look for and interpret eigensequences (in the spirit of [4, 16]).

Furthermore, it is natural to investigate how the asymptotic behavior of $\mathscr{Y}_{a, b, c, d}(x)$ depends on the asymptotic behavior of $x$, and vice versa.

\section{Appendix: Auxiliary Results on partial Bell polynomials}

The first two lemmas are known results that may be found in Comtet's book [18, Sec. 3.4 \& Sec. 3.5].

Lemma 16 (Faà di Bruno). Let $f$ and $g$ be two formal power series

$$
f(u)=\sum_{k=0}^{\infty} f_{k} \frac{u^{k}}{k !}, \quad g(t)=\sum_{m=1}^{\infty} g_{m} \frac{t^{m}}{m !},
$$

and let $h$ be the formal power series of the composition $f \circ g$,

$$
h(t)=\sum_{n=1}^{\infty} h_{n} \frac{t^{n}}{n !}=(f \circ g)(t) .
$$

Then, the coefficients $h_{n}$ are given by

$$
h_{0}=f_{0}, \quad h_{n}=\sum_{k=1}^{n} f_{k} B_{n, k}\left(g_{1}, g_{2}, \ldots, g_{n-k+1}\right) \text { for } n \geq 1 .
$$

Lemma 17. The logarithmic polynomials $L_{n}$, defined by $L_{0}=1$,

$$
\log \left(1+g_{1} t+g_{2} \frac{t^{2}}{2 !}+\cdots\right)=\sum_{n=1}^{\infty} L_{n} \frac{t^{n}}{n !},
$$

and the potential polynomials $P_{n}^{(r)}$, defined by $P_{0}^{(r)}=1$,

$$
\left(1+g_{1} t+g_{2} \frac{t^{2}}{2 !}+\cdots\right)^{r}=1+\sum_{n=1}^{\infty} P_{n}^{(r)} \frac{t^{n}}{n !},
$$

can be written for $n \geq 1$ as

$$
\begin{aligned}
L_{n} & =\sum_{k=1}^{n}(-1)^{k-1}(k-1) ! B_{n, k}\left(g_{1}, g_{2}, \ldots\right), \\
P_{n}^{(r)} & =\sum_{k=1}^{n}(r)_{k} B_{n, k}\left(g_{1}, g_{2}, \ldots\right),
\end{aligned}
$$

where $(r)_{k}=\prod_{j=0}^{k-1}(r-j)=\left(\begin{array}{l}r \\ k\end{array}\right) k !$. 
We now recall a result on partial Bell polynomials, which together with the two extensions below (Propositions 19 and 20), are needed for the proof of Theorem 1 ,

Lemma 18 ([6, Theorem 15]). Let $\alpha, \beta \in \mathbb{R}$. Given $x=\left(x_{n}\right)_{n \in \mathbb{N}}$, define $y=\left(y_{n}\right)_{n \in \mathbb{N}}$ by

$$
y_{n}=\sum_{k=1}^{n}\left(\begin{array}{c}
\alpha n+\beta k \\
k-1
\end{array}\right)(k-1) ! B_{n, k}(x) \text {. }
$$

Then, for any $\lambda \in \mathbb{C}$, we have

$$
\sum_{k=1}^{n}\left(\begin{array}{c}
\lambda \\
k-1
\end{array}\right)(k-1) ! B_{n, k}(y)=\sum_{k=1}^{n}\left(\begin{array}{c}
\alpha n+\beta k+\lambda \\
k-1
\end{array}\right)(k-1) ! B_{n, k}(x) .
$$

Proposition 19. Let $\alpha, \beta \in \mathbb{R}$. Given $x=\left(x_{n}\right)_{n \in \mathbb{N}}$, define $y=\left(y_{n}\right)_{n \in \mathbb{N}}$ by

$$
y_{n}=\sum_{k=1}^{n}\left(\begin{array}{c}
\alpha n+\beta k-1 \\
k-1
\end{array}\right)(k-1) ! B_{n, k}(x) \text {. }
$$

Then, for any $\lambda \in \mathbb{C}$, we have

$$
\sum_{k=1}^{n} \lambda^{k-1} B_{n, k}(y)=\sum_{k=1}^{n}\left(\begin{array}{c}
\alpha n+\beta k-1+\lambda \\
k-1
\end{array}\right)(k-1) ! B_{n, k}(x) .
$$

Proof. Let $f(t)=1+\sum_{n=1}^{\infty} \lambda^{n} \frac{t^{n}}{n !}$ and $g(t)=\sum_{n=1}^{\infty} y_{n} \frac{t^{n}}{n !}$. Then, Lemma 16] implies

$$
(f \circ g)(t)=1+\sum_{n=1}^{\infty} h_{n} \frac{t^{n}}{n !} \text { with } h_{n}=\sum_{k=1}^{n} \lambda^{k} B_{n, k}(y) .
$$

On the other hand, if we let $z=\left(z_{1}, z_{2}, \ldots\right)$ with

$$
z_{n}=\sum_{k=1}^{n}\left(\begin{array}{c}
\alpha n+\beta k \\
k-1
\end{array}\right)(k-1) ! B_{n, k}(x)
$$

Lemma 18 implies

$$
\begin{aligned}
& \sum_{k=1}^{n}\left(\begin{array}{c}
\lambda-1 \\
k-1
\end{array}\right)(k-1) ! B_{n, k}(z)=\sum_{k=1}^{n}\left(\begin{array}{c}
\alpha n+\beta k+\lambda-1 \\
k-1
\end{array}\right)(k-1) ! B_{n, k}(x), \\
& \sum_{k=1}^{n}\left(\begin{array}{c}
-1 \\
k-1
\end{array}\right)(k-1) ! B_{n, k}(z)=\sum_{k=1}^{n}\left(\begin{array}{c}
\alpha n+\beta k-1 \\
k-1
\end{array}\right)(k-1) ! B_{n, k}(x) .
\end{aligned}
$$

Note that the left-hand side of (24) is the logarithmic polynomial $L_{n}(z)$. Thus, if we let $Z(t)=1+\sum_{n=1}^{\infty} z_{n} \frac{t^{n}}{n !}$, (24) implies $\log (Z(t))=g(t)$. Hence $(f \circ g)(t)=e^{\lambda g(t)}=Z(t)^{\lambda}$.

On the other hand, by Lemma 17, we have

$$
\left[\frac{t^{n}}{n !}\right] Z(t)^{\lambda}=\sum_{k=1}^{n}(\lambda)_{k} B_{n, k}(z)=\sum_{k=1}^{n} \lambda\left(\begin{array}{c}
\lambda-1 \\
k-1
\end{array}\right)(k-1) ! B_{n, k}(z) .
$$

Therefore, we conclude

$$
\sum_{k=1}^{n} \lambda^{k} B_{n, k}(y)=\sum_{k=1}^{n} \lambda\left(\begin{array}{l}
\lambda-1 \\
k-1
\end{array}\right)(k-1) ! B_{n, k}(z) .
$$


Combining this identity with (23), we obtain (21).

Proposition 20. Let $\alpha, \beta, \gamma \in \mathbb{R}, \gamma \neq 0$. Given $x=\left(x_{n}\right)_{n \in \mathbb{N}}$, define $y=\left(y_{n}\right)_{n \in \mathbb{N}}$ by

$$
y_{n}=\sum_{k=1}^{n}\left(\begin{array}{c}
\alpha n+\beta k+\gamma-1 \\
k-1
\end{array}\right)(k-1) ! B_{n, k}(x) .
$$

Then, for any $\lambda \in \mathbb{C}$, we have

$$
\sum_{k=1}^{n} \gamma^{k-1}\left(\begin{array}{c}
\lambda / \gamma \\
k-1
\end{array}\right)(k-1) ! B_{n, k}(y)=\sum_{k=1}^{n}\left(\begin{array}{c}
\alpha n+\beta k+\gamma-1+\lambda \\
k-1
\end{array}\right)(k-1) ! B_{n, k}(x) .
$$

Proof. Let

$$
f(t)=(1+t)^{\frac{\lambda+\gamma}{\gamma}}=1+\sum_{n=1}^{\infty}\left(\begin{array}{c}
(\lambda+\gamma) / \gamma \\
n
\end{array}\right) n ! \frac{t^{n}}{n !}
$$

and let $g(t)=\sum_{n=1}^{\infty} \gamma y_{n} \frac{t^{n}}{n !}$. Then, by Lemma 16, we have

$$
(f \circ g)(t)=1+\sum_{n=1}^{\infty} h_{n} \frac{t^{n}}{n !} \text { with } h_{n}=\sum_{k=1}^{n}\left(\begin{array}{c}
(\lambda+\gamma) / \gamma \\
k
\end{array}\right) k ! B_{n, k}(\gamma y) .
$$

On the other hand, with $z=\left(z_{n}\right)$ defined as in (22), Lemma 18 implies

$$
\begin{aligned}
\sum_{k=1}^{n}\left(\begin{array}{c}
\lambda+\gamma-1 \\
k-1
\end{array}\right)(k-1) ! B_{n, k}(z) & =\sum_{k=1}^{n}\left(\begin{array}{c}
\alpha+\beta k+\lambda+\gamma-1 \\
k-1
\end{array}\right)(k-1) ! B_{n, k}(x), \\
\sum_{k=1}^{n} \gamma\left(\begin{array}{c}
\gamma-1 \\
k-1
\end{array}\right)(k-1) ! B_{n, k}(z) & =\gamma \sum_{k=1}^{n}\left(\begin{array}{c}
\alpha n+\beta k+\gamma-1 \\
k-1
\end{array}\right)(k-1) ! B_{n, k}(x) .
\end{aligned}
$$

Since the left-hand side of (27) is the potential polynomial $P_{n}^{(\gamma)}(z)$, this identity implies $Z(t)^{\gamma}-1=g(t)$. Finally, since $f(t)=(1+t)^{\frac{\lambda+\gamma}{\gamma}}$, we obtain $(f \circ g)(t)=Z(t)^{\lambda+\gamma}$. Thus

$$
\sum_{k=1}^{n}\left(\begin{array}{c}
(\lambda+\gamma) / \gamma \\
k
\end{array}\right) k ! B_{n, k}(\gamma y)=\sum_{k=1}^{n}(\lambda+\gamma)_{k} B_{n, k}(z)
$$

which is equivalent to

$$
\frac{\lambda+\gamma}{\gamma} \sum_{k=1}^{n}\left(\begin{array}{c}
\lambda / \gamma \\
k-1
\end{array}\right)(k-1) ! B_{n, k}(\gamma y)=(\lambda+\gamma) \sum_{k=1}^{n}\left(\begin{array}{c}
\lambda+\gamma-1 \\
k-1
\end{array}\right)(k-1) ! B_{n, k}(z) .
$$

Using (26) , and since $B_{n, k}(\gamma y)=\gamma^{k} B_{n, k}(y)$, we get (25)). 
Lemma 21. Let $\left(f_{j}\right)_{j \in \mathbb{N}}$ be the sequence given by $f_{j}=\frac{3(2 j-1) ! 2^{j}}{(j-1) !(j+2) !}$ for $j \geq 1$. For $n, k \geq 1$, we have

$$
\begin{aligned}
\frac{k !}{n !} B_{n, k}\left(1 ! f_{1}, 2 ! f_{2}, 3 ! f_{3}, \ldots\right) & \\
= & 2^{n+1}\left(\begin{array}{c}
2 n-1 \\
n-k
\end{array}\right) \frac{k}{n+k}+2^{n+1-2 k} \frac{k}{n} \sum_{i=0}^{k}(-1)^{i}\left(\begin{array}{c}
2 n+2 i-1 \\
n-1
\end{array}\right)\left(\begin{array}{c}
k-1 \\
k-i
\end{array}\right) \\
& +\sum_{j=1}^{n-1} \sum_{i=1}^{k-1} \sum_{\ell=0}^{i}(-1)^{\ell} 2^{n+1-2 i}\left(\begin{array}{c}
k \\
i
\end{array}\right)\left(\begin{array}{c}
i-1 \\
i-\ell
\end{array}\right)\left(\begin{array}{c}
2 j+2 \ell \\
j
\end{array}\right)\left(\begin{array}{c}
2 n-2 j-1 \\
n-j-k+i
\end{array}\right) \frac{i(k-i)}{(j+\ell)(n-j+k-i)} .
\end{aligned}
$$

\section{REFERENCES}

[1] J.-C. Aval, Multivariate Fuss-Catalan numbers, Discrete Math. 308 (2008), no. 20, 4660-4669.

[2] J. S. Beissinger, The enumeration of irreducible combinatorial objects, J. Combin. Theory Ser. A 38 (1985), no. 2, 143-169.

[3] E. T. Bell, Exponential polynomials, Ann. of Math. 35 (1934), 258-277.

[4] M. Bernstein and N. J. A. Sloane, Some canonical sequences of integers, Linear Algebra Appl. 226/228 (1995), 57-72.

[5] D. Birmajer, J. B. Gil, P. R. W. McNamara, and M. D. Weiner, Enumeration of colored Dyck paths via partial Bell polynomials, preprint arXiv:1602.03550, 2016.

[6] D. Birmajer, J. B. Gil, and M. D. Weiner, Some convolution identities and an inverse relation involving partial Bell polynomials, Electron. J. Combin. 19 (2012), no. 4, Paper 34, 14 pp.

[7] D. Birmajer, J. B. Gil, and M. D. Weiner, Convolutions of Tribonacci, Fuss-Catalan, and Motzkin sequences, Fibonacci Quart. 52 (2014), no. 5, 54-60.

[8] D. Birmajer, J. B. Gil, and M. D. Weiner, Colored partitions of a convex polygon by noncrossing diagonals, Discrete Math. 340 (2017), no. 4, 563-571.

[9] D. Birmajer, J. B. Gil, and M. D. Weiner, On rational Dyck paths and the enumeration of factor-free Dyck words, Discrete Appl. Math. 244 (2018), 36-43.

[10] M. T. L. Bizley, Derivation of a new formula for the number of minimal lattice paths from $(0,0)$ to $(k m, k n)$ having just $t$ contacts with the line $m y=n x$ and having no points above this line; and a proof of Grossman's formula for the number of paths which may touch but do not rise above this line, J. Inst. Actuar. 80 (1954), 55-62.

[11] M. Bóna, Exact enumeration of 1342-avoiding permutations: a close link with labeled trees and planar maps, J. Combin. Theory Ser. A 80 (1997), no. 2, 257-272.

[12] D. Callan, Counting stabilized-interval-free permutations, J. Integer Seq. 7 (2004), Article 04.1.8.

[13] D. Callan, Sets, lists and noncrossing partitions, J. Integer Seq. 11 (2008), no. 1, Article 08.1.3.

[14] P. J. Cameron, Some sequences of integers, Discrete Math. 75 (1989), no. 1-3, 89-102.

[15] C. A. Charalambides, Enumerative Combinatorics, Chapman and Hall/CRC, Boca Raton, 2002.

[16] C. Coker, A family of eigensequences, Discrete Math. 282 (2004), no. 1-3, 249-250.

[17] L. Comtet, Sur les coefficients de l'inverse de la série formelle $\sum n ! t^{n}, C$. R. Acad. Sci. Paris Sér. A-B 275 (1972), A569-A572.

[18] L. Comtet, Advanced Combinatorics: The Art of Finite and Infinite Expansions, D. Reidel Publishing Co., Dordrecht, 1974.

[19] D. Cvijović, New identities for the partial Bell polynomials, Appl. Math. Lett. 24 (2011), no. 9, 15441547.

[20] P. Duchon, On the enumeration and generation of generalized Dyck words, Discrete Math. 225 (2000), no. 1-3, 121-135.

[21] P. H. Edelman, Chain enumeration and noncrossing partitions, Discrete Math. 31 (1980), no. 2, $171-180$.

[22] S. Eger, Identities for partial Bell polynomials derived from identities for weighted integer compositions, Aequationes Math. 90 (2016), no. 2, 299-306. 
[23] A. L. L. Gao, S. Kitaev, P. B. Zhang, On pattern avoiding indecomposable permutations, Integers 18 (2018), Paper No. A2, 23 pp.

[24] H. Grosse, A. Sako, and R. Wulkenhaar, Exact solution of matricial $\Phi_{2}^{3}$ quantum field theory, Nuclear Phys. B 925 (2017), 319-347.

[25] S. Heubach, N. Y. Li, and T. Mansour, Staircase tilings and k-Catalan structures, Discrete Math. 308 (2008), no. 24, 5954-5964.

[26] D. Kreimer and K. Yeats, Diffeomorphisms of quantum fields, Math. Phys. Anal. Geom. 20 (2017), no. 2, Art. 16, 36 pp.

[27] D. Kremer, Permutations with forbidden subsequences and a generalized Schröder number, Discrete Math. 218 (2000) 121-130.

[28] V. A. Liskovets and T. R. S. Walsh, Enumeration of Eulerian and unicursal planar maps, Discrete Math. 282 (2004), 209-221.

[29] T. Mansour and Y. Sun, Bell polynomials and k-generalized Dyck paths, Discrete Appl. Math. 156 (2008), no. 12, 2279-2292.

[30] T. Mansour and Y. Sun, Dyck paths and partial Bell polynomials, Australas. J. Combin. 42 (2008), 285-297.

[31] M. Mihoubi, Bell polynomials and binomial type sequences, Discrete Math. 308 (2008), no. 12, 24502459.

[32] S. Miner and J. Pantone, Completing the structural analysis of the $2 \times 4$ permutation classes, preprint arXiv:1802.00483, 2018.

[33] J. Przytycki and A. Sikora, Polygon dissections and Euler, Fuss, Kirkman, and Cayley numbers, J. Combin. Theory Ser. A 92 (2000), no. 1, 68-76.

[34] N. J. A. Sloane, The On-Line Encyclopedia of Integer Sequences, http://oeis.org

[35] Z. Stankova, Forbidden subsequences, Discrete Math. 132 (1994), no. 1-3, 291-316.

[36] R. P. Stanley, Catalan numbers, Cambridge University Press, 2015.

[37] W. T. Tutte, A census of planar maps, Canad. J. Math. 15 (1963), 249-271.

[38] W. Wang and T. Wang, General identities on Bell polynomials, Comput. Math. Appl. 58 (2009), no. 1, $104-118$.

Nazareth College, 4245 East Ave., Rochester, NY 14618

E-mail address: abirmaj6@naz.edu

Penn State Altoona, 3000 Ivyside Park, Altoona, PA 16601

E-mail address: jgil@psu.edu

E-mail address: mdw8@psu.edu 